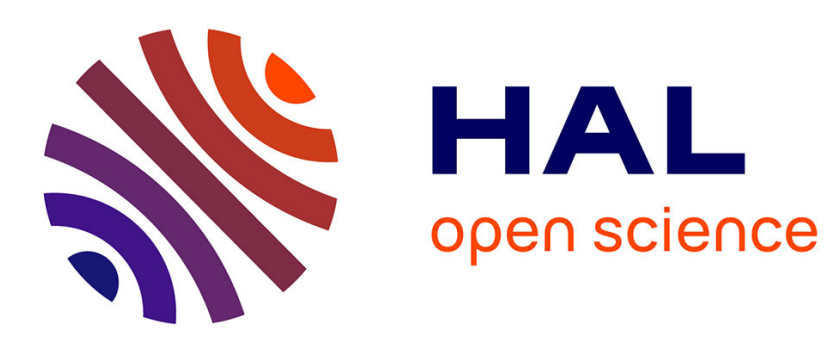

\title{
Absorption, dégradation et transport du phoséthyl-Al et de son métabolite chez la tomate (Lycopersicon esculentum Mill.)
}

\author{
Michel Luttringer, Louis de Cormis
}

\section{- To cite this version:}

Michel Luttringer, Louis de Cormis. Absorption, dégradation et transport du phoséthyl-Al et de son métabolite chez la tomate (Lycopersicon esculentum Mill.). Agronomie, 1986, 5 (5), pp.423-430. hal-00884827

\section{HAL Id: hal-00884827 \\ https://hal.science/hal-00884827}

Submitted on 1 Jan 1986

HAL is a multi-disciplinary open access archive for the deposit and dissemination of scientific research documents, whether they are published or not. The documents may come from teaching and research institutions in France or abroad, or from public or private research centers.
L'archive ouverte pluridisciplinaire HAL, est destinée au dépôt et à la diffusion de documents scientifiques de niveau recherche, publiés ou non, émanant des établissements d'enseignement et de recherche français ou étrangers, des laboratoires publics ou privés. 


\section{Absorption, dégradation et transport du phosé- thyl-Al et de son métabolite chez la tomate (Lycopersicon esculentum Mill.)}

Michel LUTTRINGER \& Louis de CORMIS

I.N.R.A., Station de Phytopharmacie, Centre de Recherches d'Avignon, F 84140 Montfavet

Trois techniques différentes (traitement de sol par arrosage, absorption pétiolaire et pulvérisation foliaire partielle après protection d'une partie de la plante) sont utilisées pour suivre le comportement du phoséthyl-Al et de son métabolite, l'acide phosphoreux.

Les résultats obtenus montrent que le phoséthyl-Al est absorbé par la plante et qu'il peut également migrer aussi bien dans le sens descendant qu'ascendant. Au cours de son déplacement, il subit une dégradation en acide phosphoreux. Ce composé, susceptible d'être lui-même absorbé en tant que tel par la plante, est aussi mobile dans les deux directions, mais tend à migrer préférentiellement vers les parties apicales.

Dans le cas des traitements du sol, des hypothèses sont avancées pour expliquer la limitation de l'absorption avec l'augmentation de la dose offerte.

Mots clés additionnels : Acide phosphoreux, systémie, absorption racinaire, absorption pétiolaire, absorption foliaire, résidus.

Additional key words : Phosphorous acid, systemic movement, root absorption, petiole absorption, foliage absorption, residues.

\section{INTRODUCTION}

Parmi les nouvelles substances antifongiques apparues au cours de la dernière décennie, les monoéthylphosphites métalliques et le phoséthyl-Al en particulier occupent une place de choix.

Ce composé, dont le nom chimique est tris-oéthylphosphonate d'aluminium, présente un grand intérêt dans la lutte contre les Phycomycètes ainsi que contre d'autres parasites (BERTRAND et al., 1977 ; Frossard et al., 1977 ; MOLOT \& BEYRIES, 1977 ; VEGH et al., 1977). Une particularité de ce produit réside dans son mode d'action (BOMPEIX et al., 1981). Contrairement aux fongicides connus jusqu'alors, le phosétyl-Al n'agit pas directement sur les champi- gnons phytopathogènes mais il est capable de stimuler et de développer les propres réactions de défense de la plante vis-à-vis de ses agresseurs (VO THI HAI et al., 1979 ; RAYNAL et al., 1980). Une des conséquences importantes de ce mode d'action serait la très faible probabilité d'apparition de souches résistantes à cette substance (BOMPEIX et al., 1981), phénomène toujours redouté avec les fongicides nouveaux (LEROUX, 1984).

Des travaux récents indiquent néanmoins l'existence d'une efficacité in vitro du phoséthyl-Al et de l'acide phosphoreux vis-à-vis de plusieurs espèces de Phytophthora (FENN \& COFFEY, 1984) et signalent même, pour la première fois, un phénomène de résistance de Phytophthora capsici à l'acide phosphoreux et au phoséthyl-Al (BOWER \& COFFEY, 1984). 
Un autre intérêt de ce produit est son caractère systémique. Différents travaux, faisant notamment appel aux techniques radio-isotopiques, ont démontré la circulation du produit dans la plante après traitement du sol ou des parties aériennes (BERTRAND et $a l$. , 1977). Ces mesures ont d'ailleurs été confirmées par des expérimentations montrant que la redistribution de la substance pouvait entraîner une protection des parties de la plante non directement traitées (BERTRAND $e t$ al., 1977 ; LAFON $e t$ al., 1977 ; CLERJEAU et al., 1981).

Des études antérieures ont montré que le phoséthyl$\mathrm{Al}$ subissait une dégradation en acide phosphoreux ou ses sels, transformation qui peut intervenir soit au niveau du sol (HASCOET, comm. pers., 1978), soit au niveau des organes traités. Toutefois, il ressort de la littérature une absence quasi complète de données sur la manière dont est absorbée, dégradée et transportée la molécule de phoséthyl-Al apportée à la plante.

Le travail présenté ci-dessous tente d'éclairer cet aspect et pourra peut-être apporter aux phytopathologistes quelques éléments de réponse concernant le pourquoi de l'efficacité ou de la non-efficacité du phoséthyl- $\mathrm{Al}$ en fonction des différents types de traitement adoptés pour la lutte.

\section{MATÉRIELS ET MÉTHODES}

\section{A. Choix et production des plantes}

Bien que la tomate ne soit pas l'un des végétaux les plus concernés par l'utilisation actuelle du phoséthyl$\mathrm{Al}$, son emploi nous a paru commode pour différentes raisons dont les principales sont la facilité d'obtention de plants en toute saison de l'année d'une part, la possibilité de standardiser les conditions de culture d'autre part, en vue d'obtenir un matériel homogène ; enfin, la morphologie du plant de tomate est bien adaptée au type de traitement que nous souhaitions effectuer.

Les graines de tomate (Lycopersicon esculentum Mill., "Tirana V, hybride F1 », obtention CLAUSE) sont mises à germer sur terreau (terreau tomates GRASSLAND, à base de sphaignes : 35 p. 100 de matière sèche, $30 \mathrm{p} .100$ de matières organique, $\mathrm{pH}\left(\mathrm{H}_{2} \mathrm{O}\right) 6$, résistivité $500 \mathrm{mMHO}$, capacité de rétention d'eau de 70 p. 100). Trois semaines à un mois après le semis selon la saison, les plantules sont repiquées sur un terreau identique dans des pots individuels. Les différentes étapes de la culture sont réalisées en serre à couverture verre. Pendant les jours les plus courts, un éclairage d'appoint (lampes PHILIPS HPL-N $400 \mathrm{~W}$ ) est utilisé pour allonger la photopériode.

\section{B. Traitement des plantes et récolte des échantillons}

\section{Traitement au sol}

Deux séries de plantes ont été traitées par apport de phoséthyl-Al sous forme d'arrosage. Une $1^{\text {re }}$ série de 80 plantes au stade 5 feuilles est utilisée pour mesurer la cinétique d'absorption de la molécule. La moitié des plantes de cette série est arrosée une fois avec $20 \mathrm{ml}$ d'une solution à $8 \mathrm{~g} / \mathrm{l}$ de phoséthyl-Al, la $2^{\mathrm{e}}$ moitié par $20 \mathrm{ml}$ d'une solution à $16 \mathrm{~g} / \mathrm{l}$. Ces traitements correspondent respectivement à des apports de 0,16 et $0,32 \mathrm{~g}$ de phoséthyl-Al par plante. Les arrosages ultérieurs sont pratiqués avec soin de façon à éviter le lessivage du sol. Les récoltes d'échantillons se font $1,2,4,8,24,48 \mathrm{~h}, 7$ et $14 \mathrm{j}$ après l'arrosage avec la matière active. A chaque fois, 5 plants sont récoltés, les tiges et pétioles séparés des limbes, les 2 fractions pesées puis conservées à $-23{ }^{\circ} \mathrm{C}$ après congélation rapide dans l'azote liquide de façon à éviter toute évolution des résidus.

Une $2^{\mathrm{e}}$ série de 40 plantes au même stade de développement est destinée à l'étude de la relation quantité offerte/quantité absorbée. A cet effet, la série est divisée en 4 lots de 10 plantes, dont chacune reçoit par arrosage respectivement 0,$16 ; 0,32 ; 0,8$ et $1,6 \mathrm{~g}$ de phoséthyl-Al. Les récoltes effectuées comme précédemment (séparation des tiges-pétioles et limbes) sont pratiquées $8 \mathrm{j}$ après traitement.

\section{Traitement par absorption pétiolaire}

20 plants de taille identique (stade 9 feuilles), sur lesquels les pousses axillaires ont été supprimées au fur et à mesure de leur apparition, sont retenus pour ce traitement. Les folioles de la $5^{\mathrm{e}}$ feuille sont coupées soigneusement au ras du pétiole à l'aide d'une lame de rasoir. Le pétiole ainsi préparé servira de voie d'absorption pour le phoséthyl-Al ou l'acide phosphoreux par trempage dans une solution de l'un ou l'autre produit contenu dans un tube à hémolyse bouché par un tampon de coton et maintenu par un ruban adhésif (fig. 1).

Parmi les 20 plantes retenues, 10 sont traitées avec une solution de phoséthyl-Al et les 10 autres avec une solution d'acide phosphoreux. Les quantités de phoséthyl-Al et d'acide phosphoreux offertes à chaque plante sont équivalentes, à savoir $7200 \mu \mathrm{g}$ de

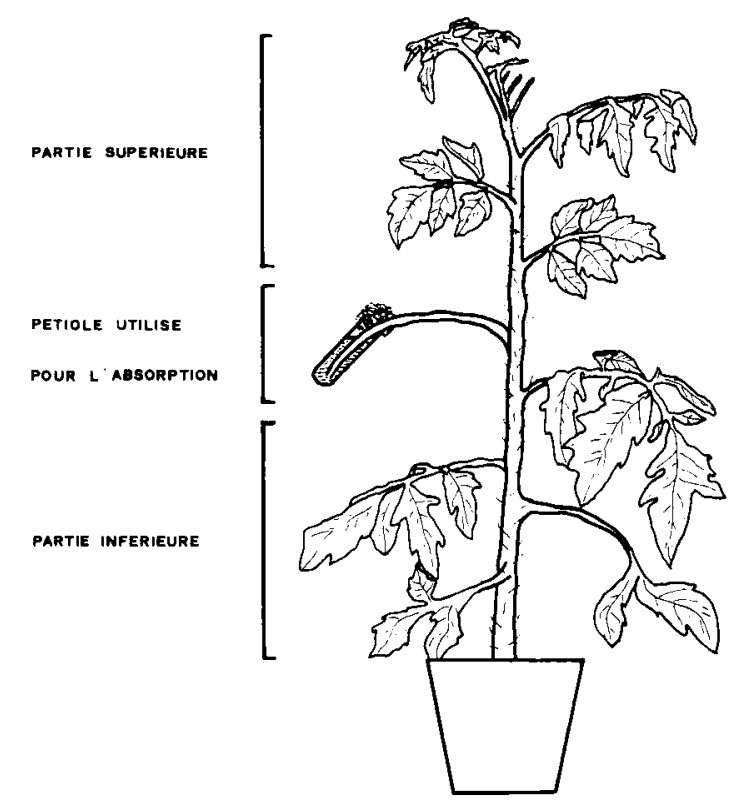

Figure 1

Préparation des plants de tomate pour l'absorption pétiolaire. Preparation of tomato plants for petiole absorption. 
phoséthyl-Al dans un cas $(5 \mathrm{ml}$ d'une solution à $1440 \mu \mathrm{g} / \mathrm{ml}$ ) et $5000 \mu \mathrm{g}$ d'acide phosphoreux dans l'autre cas $(5 \mathrm{ml}$ d'une solution à $1000 \mu \mathrm{g} / \mathrm{ml})$. Ces valeurs correspondent à des quantités identiques puisqu'une molécule de phoséthyl-Al peut donner lieu par dégradation à la formation de 3 molécules d'acide phosphoreux. Les 20 plantes de la série 1 sont récoltées au bout de $8 \mathrm{j}$ d'absorption pétiolaire, tandis que celles de la série 2 sont maintenues $15 \mathrm{j}$ avant récolte.

$\mathrm{Au}$ moment de la récolte, chaque plante est fractionnée de façon à séparer les parties situées respectivement au-dessus et au-dessous du pétiole utilisé pour la fourniture des produits étudiés. De plus, comme dans le cas précédent, une discrimination est effectuée entre les limbes d'une part, les tiges et pétioles d'autre part. Après pesage, et au cas où l'analyse de l'échantillon n'est pas réalisée immédiatement, les végétaux sont conservés au froid après congélation dans l'azote liquide.

\section{Traitement par pulvérisation foliaire partielle}

30 plants de taille comparable (stade 10 feuilles) sont utilisés pour ce traitement et préparés de la manière suivante : les plants du $1^{\text {er }}$ lot (15) sont protégés dans leur partie supérieure par un sac plastique resserré autour de la tige au-dessus de la $5^{\mathrm{e}}$ feuille, tandis que les plants du $2^{\mathrm{e}}$ lot ( 15 également) sont protégés dans leur partie inférieure par un sac plastique englobant le pot et les 5 premières feuilles. Les pots des plantes du $1^{\text {er }}$ lot sont également isolés par un sac plastique de façon à éviter un dépôt de produit sur le sol et une absorption racinaire ultérieure (fig. $2 a$ et $2 b$ )

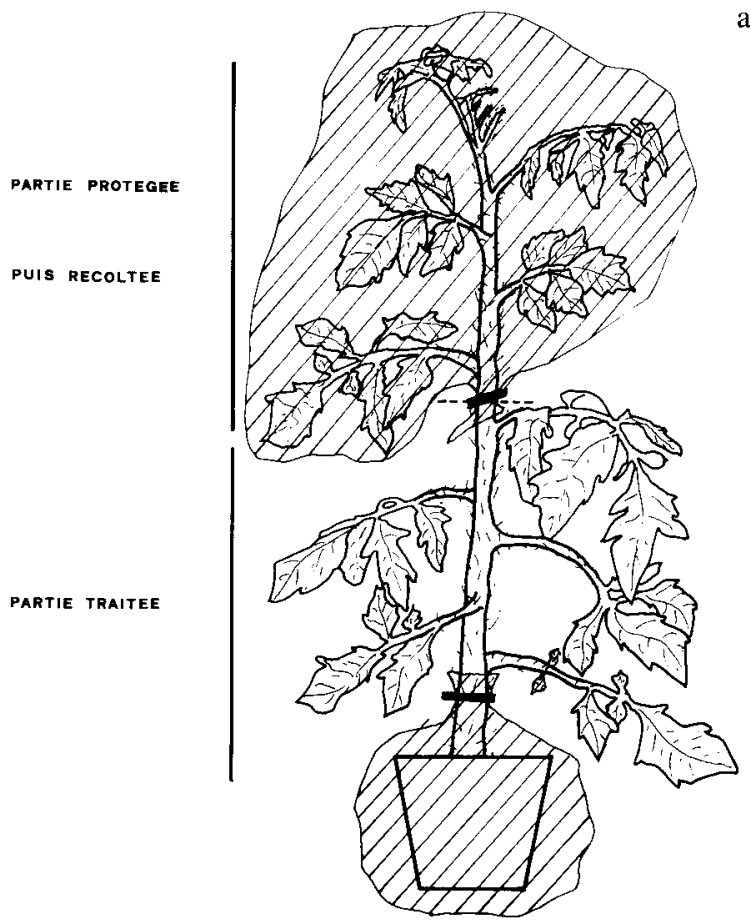

Figure 2

a. Préparation des plants de tomate pour la pulvérisation foliaire partielle. Partie basse traitée.

b. Préparation des plants de tomate pour la pulvérisation foliaire partielle. Partie haute traitée.
Le traitement des 2 lots de plantes ainsi préparées est effectué par pulvérisation d'une solution de phoséthyl-Al à $0,32 \mathrm{~g} / \mathrm{l}$ de matière active, à l'aide d'un pulvérisateur à pression préalable et jusqu'à ruissellement sur le feuillage. Après ressuyage, les protections sont enlevées avec soin afin d'éviter toute contamination des parties protégées. Les récoltes sont effectuées sur les parties n'ayant pas reçu le traitement 4,8 et $24 \mathrm{~h}$ après. Les limbes d'une part, les tiges et pétioles d'autre part, sont analysés séparément.

\section{Dosage des résidus de phoséthyl-Al et d'acide phosphoreux}

Il est effectué par chromatographie en phase gazeuse après méthylation des extraits. Une prise d'essai de $20 \mathrm{~g}$ de matière fraîche est broyée en présence d'une solution d'acide sulfurique (1 p. 100 poids/volume) à l'aide d'un omni-mixer SORVALL pendant $2 \mathrm{mn}$. Les extraits sont ensuite centrifugés, puis filtrés et amenés à un volume de $100 \mathrm{ml}$ avec de l'eau distillée. Une partie aliquote de cette solution $(1 \mathrm{ml})$ est méthylée en présence d'acétone et d'acide acétique par le diazométhane obtenu par action d'une solution à environ 20 p. 100 (poids/volume) de Nméthyl-N-tosylnitrosamide dans l'éther monométhylique de l'éthylène glycol sur une solution d'hydroxyde de potassium à $10 \mathrm{p} .100$ dans le méthanol ou l'éther monométhylique de l'éthylène glycol. Après méthylation et destruction de l'excès de diazométhane par addition d'une goutte d'acide acétique, la solution est volumée à $10 \mathrm{ml}$ dans l'acétone puis injectée dans le chromatographe (GIRDEL, série 30 ).

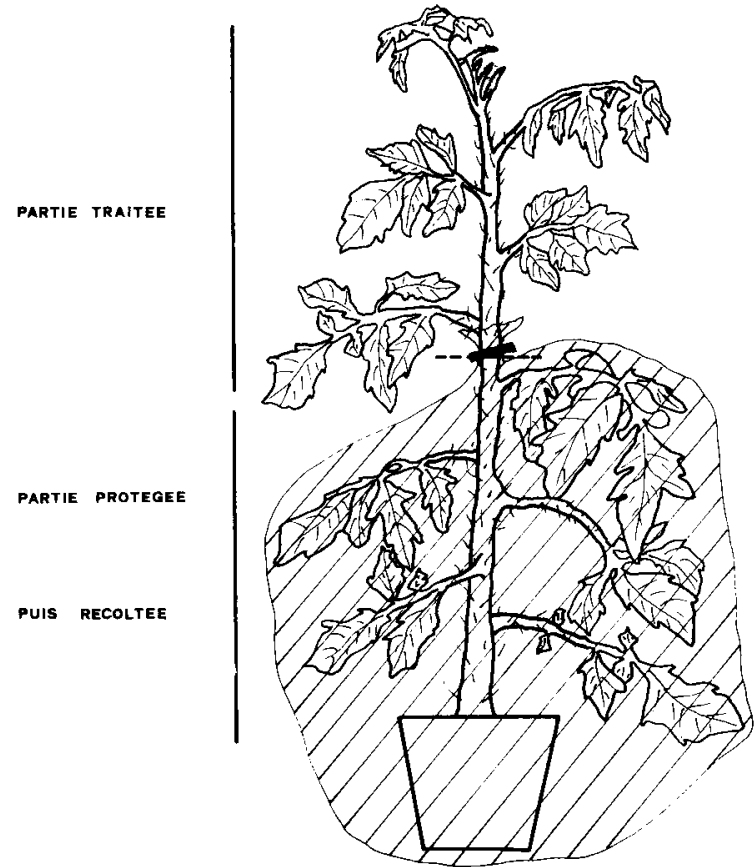

b

a. Preparation of tomato plants for partial spraying of foliage. Lower part treated.

b. Preparation of tomato plants for partial spraying of foliage. Upper part treated. 
La colonne utilisée pour la séparation présente les caractéristiques suivantes : colonne de verre de longueur 4 pieds environ, remplie de CARBOWAX $20 \mathrm{M}$ à 10 p. 100 sur GaS CHROM Q 100/120 mesh. La température du four est maintenue à $155^{\circ} \mathrm{C}$. Les températures de l'injecteur et du détecteur sont respectivement fixées à 210 et $250{ }^{\circ} \mathrm{C}$. Les résidus de phoséthyl$\mathrm{Al}$ et d'acide phosphoreux, transformés en éthylméthylphosphite et diméthylphosphite lors de la méthylation sont détectés par un détecteur thermoionique (spécifique du phosphore).

Dans les conditions opératoires fixées, les temps de rétention respectifs du diméthylphosphite et de l'éthylméthylphosphite sont de 4,8 et 5,6 mn. Les concentrations des échantillons sont déterminées par référence à des solutions-étalons de phoséthyl-Al et d'acide phosphoreux méthylées dans les mêmes conditions. Les conditions analytiques décrites ci-dessus permettent d'atteindre pour chaque composé une limite de détection de $0,2 \mu \mathrm{g} / \mathrm{g}$.

\section{RÉSULTATS}

\section{A. Traitement par arrosage}

\section{Cinétique d'absorption}

Les résultats d'analyse de phoséthyl-Al et d'acide phosphoreux sont regroupés dans le tableau 1. Une première constatation concerne l'existence de résidus de phoséthyl-Al dans les 2 premiers prélèvements (1 et $2 \mathrm{~h}$ après traitement).

L'arrosage à $0,16 \mathrm{~g} /$ pot provoque un résidu dans les limbes uniquement, tandis que l'arrosage à une dose double induit la présence de résidus également dans les tiges et pétioles. En revanche, dans tous les prélèvements postérieurs à $2 \mathrm{~h}$, on ne retrouve plus de phoséthyl-Al.

L'évolution des résidus d'acide phosphoreux est assez comparable dans les 2 séries : faibles dans les $4 \mathrm{~h}$ qui suivent le traitement, ils augmentent régulièrement jusqu'à $7 \mathrm{j}$ dans les tiges et pétioles. Les niveaux de résidus restent du même ordre de grandeur entre les prélèvements de $7 \mathrm{j}$ et $14 \mathrm{j}$. En revanche, dans les limbes, l'augmentation des concentrations est constante depuis le prélèvement de $8 \mathrm{~h}$ jusqu'à celui de $14 \mathrm{j}$.

\section{Relation dose offerte/quantité absorbée}

Les variations des concentrations en acide phosphoreux sont illustrées par la figure 3 . Pour cette série de mesures effectuées $8 \mathrm{j}$ après le traitement du sol, aucun résidu de phoséthyl-Al n'a été détecté, ce qui confirme les résultats précédents (cinétique d'absorption). Les concentrations en acide phosphoreux dans les différentes parties de la plante varient dans le même sens que les doses apportées au sol, sans toutefois leur être directement proportionnelles. Il semble, au contraire, que ces concentrations tendent vers un palier.

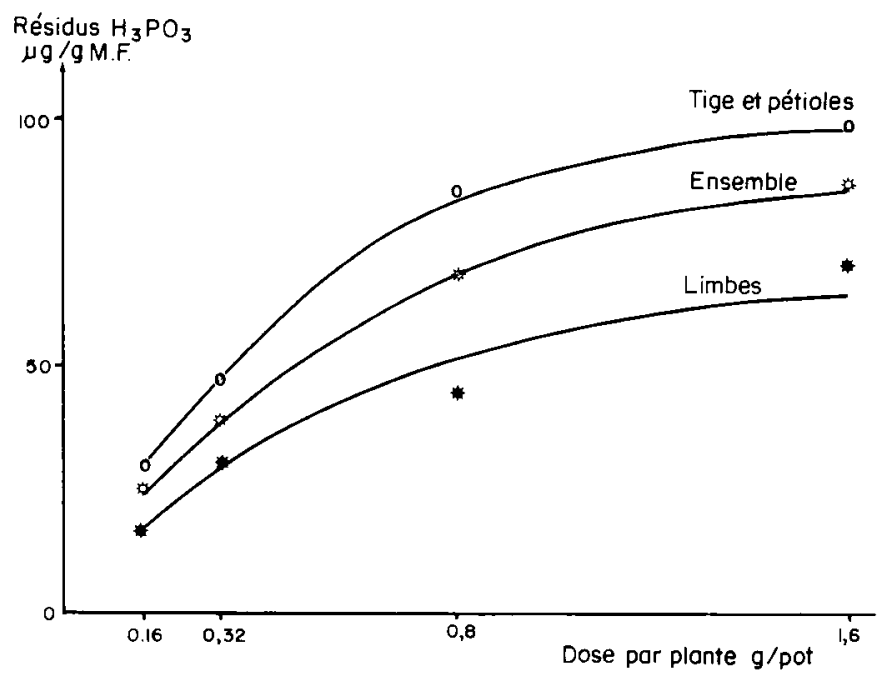

Figure 3

Résidus d'acide phosphoreux dans les plantes $(\mu \mathrm{g} / \mathrm{g}$ de matière fratche) $8 j$ après traitement par arrosage avec le phoséthyl-Al.

Phosphorous acid residues in plants ( $\mu \mathrm{g} / \mathrm{g}$ fresh weight) eight days after watering treatment with fosetyl-Al.

TABLEAU

Résidus de phoséthyl-Al et d'acide phosphoreux $(\mu \mathrm{g} / \mathrm{g}$ de matière fraîche) dans les plantes après traitement par arrosage. Fosetyl-Al and phosphorous acid residues ( $\mu \mathrm{g} / \mathrm{g}$, fresh weight) in plants after watering treatment.

\begin{tabular}{|c|c|c|c|c|c|c|c|c|}
\hline & \multicolumn{4}{|c|}{$0,16 \mathrm{~g} / \mathrm{pot}$} & \multicolumn{4}{|c|}{$0,32 \mathrm{~g} /$ pot } \\
\hline & \multicolumn{2}{|c|}{ Tiges et pétioles } & \multicolumn{2}{|c|}{ Limbes } & \multicolumn{2}{|c|}{ Tiges et pétioles } & \multicolumn{2}{|c|}{ Limbes } \\
\hline & Phos. Al & Ac. Phos. & Phos. Al & Ac. Phos. & Phos. Al & Ac. Phos. & Phos. Al & Ac. Phos. \\
\hline $1 \mathrm{~h}$ & $\mathrm{ND}^{+}$ & 1,5 & 1,4 & 1,4 & 0,8 & 2,2 & 3,5 & 1,5 \\
\hline $2 \mathrm{~h}$ & ND & 3,0 & 3,6 & 2,3 & 0,8 & 1,2 & 1,3 & 1,4 \\
\hline $4 \mathrm{~h}$ & ND & 2,9 & ND & 1,8 & ND & 2,7 & ND & 2,5 \\
\hline $8 \mathrm{~h}$ & ND & 7,8 & ND & 6,7 & ND & 11,9 & ND & 8,5 \\
\hline $24 \mathrm{~h}$ & ND & 19,0 & ND & 7,1 & ND & 24,1 & ND & 9,1 \\
\hline $48 \mathrm{~h}$ & ND & 27,6 & ND & 18,2 & ND & 30,1 & ND & 16,3 \\
\hline $7 \mathrm{j}$ & ND & 101,0 & ND & 43,2 & ND & 156,2 & ND & 51,7 \\
\hline $14 \mathrm{j}$ & ND & 100,0 & ND & 93,0 & ND & 149,1 & ND & 132,8 \\
\hline
\end{tabular}

\footnotetext{
+ ND : non détecté à la limite de $0,2 \mu \mathrm{g} / \mathrm{g}$.
} 
Comme dans l'essai précédent, les tiges et pétioles renferment davantage d'acide phosphoreux que les limbes, le rapport des concentrations étant compris entre 1,5 et 2 .

On notera également que les niveaux de résidus ne sont pas comparables avec ceux de l'essai précédent. Cette variation importante peut être attribuée aux conditions climatiques régnant lors des 2 essais qui se sont déroulés à des périodes différentes de l'année. L'état physiologique des 2 séries de plantes utilisées se répercutant vraisemblablement sur leurs capacités d'absorption, il n'est pas possible de comparer quantitativement les 2 essais, seules les variations enregistrées au cours de l'un ou l'autre étant interprétables.

\section{B. Traitement par absorption pétiolaire}

Les résultats des analyses de résidus en fonction de la date de récolte sont regroupés sur le tableau 2 (série 1: $8 \mathrm{j}$ d'absorption pétiolaire ; série $2: 15 \mathrm{j}$ d'absorption pétiolaire). Avant d'examiner les résultats dans le détail, il convient de rapporter l'observation suivante effectuée au cours de la manipulation : si l'absorption pétiolaire de la solution d'acide phosphoreux est apparue assez rapide (quelques jours suffisent pour que la totalité des $5 \mathrm{ml}$ soit absorbée), elle est beaucoup plus lente, voire incomplète, avec la solution de phoséthyl-Al puisque, même après $15 \mathrm{j}$, il reste encore une fraction des $5 \mathrm{ml}$ non utilisée par la plante. Notons par ailleurs qu'aucun signe de phytotoxicité n'est apparu sur les plantes au cours de ces différents traitements.

Concernant les résultats des analyses, une remarque commune aux 2 séries s'impose : on note en effet, dans les 2 cas, la présence de résidus de phoséthyl-Al non transformé dans les plantes soumises à l'absorption de la solution de phoséthyl-Al ; les concentrations mesurées sont d'ailleurs très voisines les unes des autres tant dans les parties inférieures que dans les parties supérieures des plantes. Les chiffres obtenus pour la série 1 montrent toutefois des concentrations plus élevées dans les limbes que dans les tiges et pétioles; ils ne sont pas confirmés par les résultats de la série 2.

Les concentrations en acide phosphoreux sont, pour leur part, très dépendantes du type de traitement subi par les plantes : pour la série 1 , dans le cas du traite- ment avec le phoséthyl-Al, les teneurs résiduelles en phosphites sont comparables à la fois dans les tiges et pétioles et les limbes des parties inférieure et supérieure au point d'absorption. Au contraire, le traitement effectué avec l'acide phosphoreux conduit à des concentrations plus variables. Faible dans les tiges et pétioles de la partie inférieure, la teneur en acide phosphoreux est environ 3 fois plus importante dans les limbes correspondants et du même ordre de grandeur, tant dans les tiges et pétioles que dans les limbes de la partie supérieure.

Les résultats obtenus dans la $2^{\mathrm{e}}$ série se différencient nettement de ceux de la $1^{\text {re }}$ série. En effet, avec le traitement phoséthyl-Al, les concentrations en acide phosphoreux sont globalement plus importantes que dans la série 1 avec une prédominance dans les tiges et pétioles. Le traitement avec l'acide phosphoreux conduit à des concentrations plus faibles que pour la série 1 , avec une différence marquée entre l'ensemble tiges-pétioles et les limbes, en particulier pour la partie supérieure des plantes.

\section{Traitement par pulvérisation foliaire partielle}

Les résultats des analyses de résidus de phoséthyl-Al et d'acide phosphoreux sont regroupés sur le tableau 3. Il convient de noter que, lors de cette manipulation, seules ont été récoltées les parties des plantes qui avaient été préalablement protégées; en effet, l'analyse des parties sur lesquelles ont été effectuées les pulvérisations n'apporterait aucun renseignement utilisable du fait de la prise en compte lors de cette analyse du dépôt de matière active à la surface des organes. Une analyse pourrait être envisagée après élimination du dépôt superficiel, à condition toutefois de parfaitement maîtriser son efficacité (un tel lavage doit faire disparaître totalement le produit déposé à la surface du végétal sans extraire une partie du résidu déjà absorbé par la plante).

Deux remarques sont à faire concernant les résultats du tableau 3. En premier lieu, il faut voir que les résidus de phoséthyl-Al se retrouvent aussi bien dans les parties inférieure que supérieure. En second lieu, on remarquera les variations en fonction du temps qui indiquent une décroissance globale des résidus nettement plus marquée dans les limbes que dans les tiges et pétioles.

TABLEAU 2

Résidus de phoséthyl-Al et d'acide phosphoreux ( $\mu \mathrm{g} / \mathrm{g}$ de matière fraîche) dans les différentes parties de la plante après $8 j$ et $15 j$ d'absorption pétiolaire.

Fosetyl-Al and phosphorous acid residues ( $\mu \mathrm{g} / \mathrm{g}$ fresh weight) in various parts of plants after eight days and fifteen days of petiole absorption.

\begin{tabular}{|c|c|c|c|c|c|c|c|c|c|}
\hline & & \multicolumn{4}{|c|}{ Partie inférieure } & \multicolumn{4}{|c|}{ Partie supérieure } \\
\hline & & \multicolumn{2}{|c|}{ Tiges et pétioles } & \multicolumn{2}{|c|}{ Limbes } & \multicolumn{2}{|c|}{ Tiges et pétioles } & \multicolumn{2}{|c|}{ Limbes } \\
\hline & & $8 \mathrm{j}$ & $15 \mathrm{j}$ & $8 \mathrm{j}$ & $15 \mathrm{j}$ & $8 \mathrm{j}$ & $15 \mathrm{j}$ & $8 \mathrm{j}$ & $15 \mathrm{j}$ \\
\hline Traitement & Phos. Al & 0,2 & 0,5 & 0,5 & 0,5 & 0,2 & 0,7 & 0,4 & 0,3 \\
\hline Phos. Al & Ac. Phos. & 3,8 & 4,8 & 3,1 & 3,4 & 3,4 & 4,5 & 3,4 & 3,3 \\
\hline $\begin{array}{l}\text { Traitement } \\
\text { Ac. Phos. }\end{array}$ & Ac. Phos. & 3,2 & 3,8 & 10,2 & 5,4 & 10,8 & 5,4 & 12,9 & 9,0 \\
\hline
\end{tabular}


TABLEAU 3

Résidus de phoséthyl-Al et d'acide phosphoreux ( $\mu \mathrm{g} / \mathrm{g}$ de matière fraiche) après traitement par pulvérisation partielle avec une solution à $0,4 \mathrm{~g} / \mathrm{l}$ de phoséthyl-Al.

Fosetyl-Al and phosphorous acid residues ( $\mu \mathrm{g} / \mathrm{g}$ fresh weight)

after partial spraying foliage treatment with a solution containing $0.4 \mathrm{~g} / \mathrm{l}$ fosetyl-Al.

\begin{tabular}{|c|c|c|c|c|c|c|c|c|}
\hline & \multicolumn{4}{|c|}{ Traitement de la partie supérieure } & \multicolumn{4}{|c|}{ Traitement de la partie inférieure } \\
\hline & \multicolumn{4}{|c|}{ Partie inférieure récoltée } & \multicolumn{4}{|c|}{ Partie supérieure récoltée } \\
\hline & \multicolumn{2}{|c|}{ Tiges et pétioles } & \multicolumn{2}{|c|}{ Limbes } & \multicolumn{2}{|c|}{ Tiges et pétioles } & \multicolumn{2}{|c|}{ Limbes } \\
\hline & Phos. Al & Ac. Phos. & Phos. Al & Ac. Phos. & Phos. Al & Ac. Phos. & Phos. Al & Ac. Phos \\
\hline $4 \mathrm{~h}$ & 1,4 & 4,8 & 3,3 & 3,1 & 1,6 & 3,6 & 3,2 & 3,3 \\
\hline $8 \mathrm{~h}$ & 1,1 & 2,8 & 2,7 & 9,8 & 1,2 & 5,4 & 2,8 & 6,3 \\
\hline $24 \mathrm{~h}$ & 1,0 & 2,9 & 1,6 & 3,1 & 1,4 & 3,1 & 1,9 & 5,6 \\
\hline
\end{tabular}

Les résultats se rapportant à l'acide phosphoreux sont différents des précédents en ce sens que les variations constatées ne peuvent être directement corrélées aux variations de concentrations en phoséthyl-Al. Les concentrations en acide phosphoreux sont plus importantes dans les limbes que dans les tiges et pétioles, excepté pour le prélèvement le plus rapproché du traitement. Un maximum très marqué apparaît dans les limbes de la partie inférieure lors du prélèvement effectué $8 \mathrm{~h}$ après pulvérisation de la partie haute, suivi d'une baisse sensible au bout de $24 \mathrm{~h}$. L'évolution des concentrations dans les parties apicales est tout à fait similaire, avec des amplitudes toutefois moins prononcées.

\section{DISCUSSION ET CONCLUSION}

L'étude de l'absorption après traitement du sol par arrosage avec une solution de phoséthyl-Al nous a fourni un certain nombre de données intéressantes. En premier lieu, la plante absorbe bien le phoséthyl-Al dans les $1^{\text {res }}$ heures qui suivent le traitement. La présence d'acide phosphoreux devient indiscutable à partir du prélèvement effectué $8 \mathrm{~h}$ après le traitement et le maximum de concentration est atteint au bout de $7 \mathrm{j}$, bien que la croissance de la plante, donc la dilution qui s'ensuit, masque partiellement la quantité réellement absorbée. Par ailleurs, dans le cas de plantes à croissance plus lente (Capsicum annuum L.), l'augmentation des concentrations en acide phosphoreux se poursuit pendant un mois. Il peut être intéressant de rapprocher ces résultats de ceux obtenus par d'autres chercheurs qui constatent une inefficacité du phoséthyl-Al au niveau de la protection de feuilles de plants de poivron traités par arrosage et artificiellement contaminés par Phytophthora capsici Leon. $24 \mathrm{~h}$ seulement après le dernier arrosage (CLERJEAU \& BEYRIES, 1977). Dans une expérience similaire, YUSUF (1981) note l'inefficacité du phosétyl-Al sur l'anthracnose du haricot (Colletotrichum lindemuthianum Sacc. \& Magne) après arrosage du terreau un jour seulement avant l'inoculation. Il est vraisemblable que, dans ces 2 cas, la migration du phoséthyl-Al et surtout de l'acide phosphoreux n'a pas encore été très importante.

Par ailleurs, la concentration en phosphites est toujours plus élevée dans les tiges et pétioles que dans les limbes. On peut donc penser que la migration du métabolite est en cours, sans toutefois rejeter l'hypothèse d'une circulation "à double sens " de l'acide phosphoreux, hypothèse qu'une simple analyse de la partie aérienne de la plante ne peut vérifier. Une étude visant à déterminer les résidus dans les courants ascendant et descendant a été partiellement réalisée : l'analyse du liquide de guttation des feuilles de tomate dont les racines avaient absorbé une dose de phoséthyl-Al, d'une part, et, d'autre part, celle de l'exsudat libérien de feuilles de fève ayant préalablement reçu une application de l'antifongique ont mis en évidence cette systémie ascendante et descendante du phoséthyl-Al et de son métabolite (LECONTE, 1984).

Au cours des 2 manipulations par traitement du sol, des doses différentes de phoséthyl-Al ont été mises en œuvre. Bien que la quantité de métabolite retrouvée dans la plante augmente avec la dose de traitement, il ne semble pas exister de relation linéaire entre elles. Plusieurs explications peuvent être envisagées :

- soit un blocage du phoséthyl-Al ou de l'acide phosphoreux par un ou plusieurs constituants du sol, - soit une limitation de l'absorption par la plante elle-même, par exemple par un blocage ou une accumulation au niveau des racines.

A ce stade de la discussion, quelques remarques s'imposent: l'absorption pétiolaire n'est pas un moyen courant pour étudier l'absorption, le transport et, le cas échéant, la dégradation d'une substance agropharmaceutique. La majorité des techniques fait appel à des traitements de sol ou à des applications sur les parties aériennes. Il nous est apparu important d'exclure l'apport au sol ; en effet, l'absorption racinaire dépend, en plus de l'aptitude de la plante à absorber le produit, des phénomènes souvent antagonistes qui ont lieu dans le sol, tels qu'un blocage partiel ou total, une dégradation et/ou un lessivage. Si donc la " composante sol " ne peut être écartée dans le cadre d'une étude globale, l'absorption pétiolaire, bien que peu naturelle, permet de mettre à la disposition de la plante une certaine quantité de substance à un niveau préalablement choisi et sans intermédiaire. 
La $2^{\mathrm{e}}$ technique employée (pulvérisation après protection partielle) se rapproche davantage des moyens traditionnels et autorise, en outre, l'étude dans de bonnes conditions du transport d'un produit quelconque.

La technique de l'absorption pétiolaire permet de constater la présence de résidus de phoséthyl-Al non transformé dans la plante. Le phoséthyl-Al peut donc non seulement pénétrer dans la plante, mais aussi s'y déplacer, tant dans le sens ascendant que descendant. Ces résultats confirment ceux obtenus après traitement du sol où les résidus de phoséthyl-Al étaient détectés dans les $1^{\text {res }}$ heures suivant le traitement. La disparition de ces résidus après $4 \mathrm{~h}$ peut être attribuée à la dégradation du phoséthyl-Al dans le sol (y compris sa transformation en acide phosphoreux), donc à la suppression de la source disponible pour la plante. $\mathrm{Au}$ contraire, le traitement par absorption pétiolaire présente un caractère continu du fait de la lente absorption de la solution de phoséthyl-Al signalée plus haut, ce qui explique la présence de résidus $15 \mathrm{j}$ encore après le début du traitement.

Les chiffres concernant les résidus d'acide phosphoreux sont naturellement plus intéressants dans la mesure où il s'agit du métabolite principal du phoséthyl-Al qui présente une réelle activité antifongique. La comparaison des chiffres obtenus lors des traitements par absorption pétiolaire soit avec le phoséthyl-Al soit avec l'acide phosphoreux montre que dans le $1^{\text {er }}$ cas, une répartition quasi identique de l'acide phosphoreux s'établit entre les parties inférieure et supérieure au point d'absorption. Au contraire, le traitement avec l'acide phosphoreux provoque une accumulation plus importante dans la partie inférieure avec une prédominance dans les limbes par rapport aux tiges et pétioles.

On peut donc proposer pour le phoséthyl-Al et son métabolite le cheminement suivant : absorption par le pétiole, migration préférentielle vers les parties supérieures pour l'acide phosphoreux provenant de la dégradation du phoséthyl-Al au fur et à mesure de son déplacement qui semble identique tant vers la base que vers l'apex.

Pour le traitement à l'acide phosphoreux, on retrouve globalement moins de résidu au bout de $15 \mathrm{j}$ qu'au bout de $8 \mathrm{j}$. Deux hypothèses peuvent être formulées : la $1^{\text {re }}$ concerne une éventuelle excrétion raci- naire qui serait la conséquence ultime du transport descendant, la $2^{\mathrm{e}}$ fait appel aux capacités de métabolisation de la plante transformant les phosphites en phosphates. Des expériences sont en cours pour vérifier le bien-fondé de ces hypothèses.

Les traitements effectués par pulvérisation foliaire partielle viennent confirmer, pour certains points, les résultats obtenus par absorption pétiolaire. C'est le cas notamment pour l'absorption et la migration du phoséthyl-Al. Les résidus d'acide phosphoreux, plus élevés dans les tiges et pétioles que dans les limbes $4 \mathrm{~h}$ après le traitement, laissent supposer que le transport est en cours et qu'une fraction importante du phoséthyl-Al est aussitôt dégradée en acide phosphoreux, le reste étant transporté vers les limbes où la dégradation interviendra ultérieurement. Au prélèvement effectué $8 \mathrm{~h}$ après le traitement, on note une élévation importante de la concentration en acide phosphoreux dans les limbes et surtout dans ceux des plantes traitées par la partie supérieure; en revanche, après $24 \mathrm{~h}$, il y a une baisse sensible dans les limbes de la partie inférieure et peu de fluctuations dans ceux de la partie supérieure.

Les 2 hypothèses avancées plus haut, à propos de la diminution au cours du temps de la concentration en acide phosphoreux dans les plantes, pourraient aussi être évoquées pour l'explication des variations mesurées dans ce cas.

On rapprochera utilement de ces remarques celles effectuées par CLERJEAU et al. (1981) à propos du délai nécessaire (plus de $2 \mathrm{j}$ ) à une bonne efficacité du phoséthyl-Al appliqué sur feuilles de vigne contaminées ensuite avec Plasmopara viticola (Berk. \& Curt.) Berl. \& de Toni.

En définitive, les différentes analyses effectuées au cours de ce travail ne donnent qu'une " photographie " des résidus présents à l'instant du prélèvement et ne permettront que des hypothèses sur les voies de transport utilisées par le phoséthyl-Al et son métabolite. Des études mettant en œuvre des techniques plus appropriées seront nécessaires pour déterminer avec précision le cheminement et le devenir de ces 2 substances.

Reçu le 6 septembre 1984. Accepté le 27 décembre 1984.

\section{RÉFÉRENCES BIBLIOGRAPHIQUES}

\begin{abstract}
Bertrand A., Ducret J., Debourge J. C., Horrière D., 1977. Etude des propriétés d'une nouvelle famille de fongicides: les monoéthylphosphites métalliques. Caractéristiques physicochimiques et propriétés biologiques. Phytiatr. Phytopharm., 26, 3-18.
\end{abstract}

Bompeix G., Fettouche F., Saindrenan P., 1981. Mode d'action du phoséthyl-Al. Phytiatr. Phytopharm., 30, 257-272.

Bower L. A., Coffey M. D., 1984. Development of resistance to phosphorous acid in Phytophthora capsici. Phytopathology, 74, (7), A 169.

Clerjeau M., Beyries A., 1977. Etude comparée de l'action préventive et du pouvoir systémique de quelques fongicides nouveaux (phosphites, prothiocarbe, pyroxychlore) sur poivron vis-à-vis de Phytophthora capsici Leon. Phytiatr. Phytopharm., 26, 73-84.
Clerjeau M., Lafon R., Bugaret Y., 1981. Etudes sur les propriétés et le mode d'action des nouveaux fongicides antimildiou chez la vigne (cymoxanil, métalaxyl, milfurame, phoséthyl-Al). Phytiatr. Phytopharm., 30, 215-234.

Fenn M. E., Coffey M. D., 1984. Studies on the in vitro and in vivo antifungal activity of fosetyl-Al and phosphorous acid. Phytopathology., 74 (7), A 515.

Frossard P., Haury A., Laville E., 1977. Résultats préliminaires concernant l'activité de l'éthylphosphite d'aluminium (LS 74783) sur les maladies à Phytophthora des agrumes, de l'avocatier et de l'ananas. Phytiatr. Phytopharm., 26, 55-62.

Lafon R., Bugaret Y., Bulit J., 1977. Nouvelles perspectives de lutte contre le mildiou de la vigne (Plasmopara viticola (B.C.) Berl. 
et de Toni) avec un fongicide systémique, l'éthylphosphite d'aluminium. Phytiatr. Phytopharm., 26, 19-40.

Leconte F., 1984. Introduction à l'étude de la migration du phoséthyl-Al dans la plante. DEA, Univ. Poitiers.

Leroux P., 1984. La résistance des champignons phytopathogènes aux fongicides : conséquence de l'intensification de la lutte chimique ? Déf. Vég., 227, 135-145.

Molot P. M., Beyries A., 1977. Etude comparée de quelques fongicides nouveaux (prothiocarbe, pyroxychlore, phosphites) dans la lutte contre Phytophthora cactorum Leb. \& Cohn du fraisier. Phytiatr. Phytopharm., 26, 63-72.

Raynal G., Ravisé A., Bompeix G., 1980. Action du tris-o-éthylphosphonate d'aluminium (phoséthyl-Al) sur la pathogénie de Plas- mapora viticola et sur la stimulation des réactions de défense de la vigne. Ann. Phytopathol., 12 (3), 163-175.

Vegh I., Baillot F., Roy J., 1977. Etude de l'activité de l'éthylphosphite d'aluminium (LS 74783) vis-à-vis de Phytophthora cinnamomi Rands, agent du dépérissement des arbustes d'ornement. Phytiatr. Phytopharm., 26, 85-96.

Vo Thi Hai, Bompeix G., Ravisé A., 1979. Rôle du tris-oéthylphosphonate d'aluminium (phoséthyl-Al) dans la stimulation des réactions de défense des tissus de tomate contre le Phytophthora capsici. C. R. Acad. Sci., Paris, 288, 1171-1174.

Yusuf A. J., 1981. Etude de l'effet du phoséthyl-aluminium (Aliet$\mathrm{te}^{\mathrm{R}}$ ) vis-à-vis de l'anthracnose de haricot. Parasitica, 37, (1), 3-13. 